\title{
STRAINS IN AN INFLATED RUBBER SHEET
}

\author{
P. H. MotT, * C. M. Roland \\ CHEMISTRY Division \\ NAVAL ReSEARCh LABORATORY, WASHington, DC 20375 \\ S. E. HASSAN \\ Propulsion, Hydrodynamics and SiLENCING Division \\ Naval Undersea Warfare CENTER, NewPort, RI 02841
}

\begin{abstract}
A technique to measure the strain distribution of an inflated membrane is described, and applied to natural rubber inflated to pole biaxial strains of $12 \%, 26 \%$, and $33 \%$. The radial strain was found to be nearly constant over the entire surface, while the circumferential strain falls to zero at the edge. Thus, biaxial deformation is limited to a narrow region in the vicinity of the pole. These results are quite different from (the very limited) data published previously. The stressstrain behavior was also measured in uniaxial tension, and the strain distribution of the inflated membrane calculated using finite elements. The experiment results and the modeling were in good agreement.
\end{abstract}

\section{INTRODUCTION}

To predict the service life of a rubber article requires knowledge of the mode, as well as the magnitude, of the deformations. The strains reflect the interplay between loading and geometry, and can lead to failure at positions away from the region of maximum strain. For a circular, inflated elastic membrane, clamped at the edge, the local deformations are a complex function of radius, from biaxial at the pole to planar extension (pure shear) at the outer radius. The details of the transition between these two extremes depend on the strain energy function. ${ }^{1}$

Since inflation and biaxial straining are common in many applications of elastomers, a quantitative understanding of this mode of deformation has obvious value. Recently, interest in the strain distribution in inflating membranes has extended to human pathology, e.g., aneurysms. ${ }^{2,3}$ However, investigations of the strain distribution in membranes are rare, ${ }^{4}$ and studies of crack growth in biaxial deformation yield conflicting conclusions. ${ }^{5,6}$ The present study was motivated by the development of an inflated rubber disk as an ejection system for torpedoes on US Navy Virginia-class submarines. ${ }^{7-9}$ During fatigue testing, the failure properties were found to be dependent on the size of the disk. Such a scaling effect indicates that biaxial deformation at the pole, which is the largest strain, does not necessarily govern the failure. ${ }^{10}$

\section{BACKGROUND}

Inflation of a flat circular elastomeric membrane has become a classical problem in the mechanics of incompressible materials. The strain distributions in the circumferential (latitude), $\varepsilon_{\mathrm{C}}$, and radial (longitude), $\varepsilon_{\mathrm{R}}$, directions were measured as a function of position by Treloar. ${ }^{4}$ These results, obtained on a gum natural rubber, are reproduced in Figure 1. The abscissa of the figure ("degrees from pole") was defined as the angle between the pole and the latitude line at the center of curvature of the inflating sheet. Unfortunately, since the position of the center of curvature was not specified, the original radial position data cannot be unambiguously recovered. A qualitative relationship between these abscissa values and the normalized radial positions can be inferred: The pole (center), where the circumferential $\varepsilon_{C}$ and the radial $\varepsilon_{R}$ strains are always

* Corresponding author. Ph: 202-767-1720; Fax: 202-767-0594; email: phm@xbt.nrl.navy.mil 
equal, is at zero degrees, while the outer radius corresponds to where $\varepsilon_{\mathrm{C}}$ falls to zero.

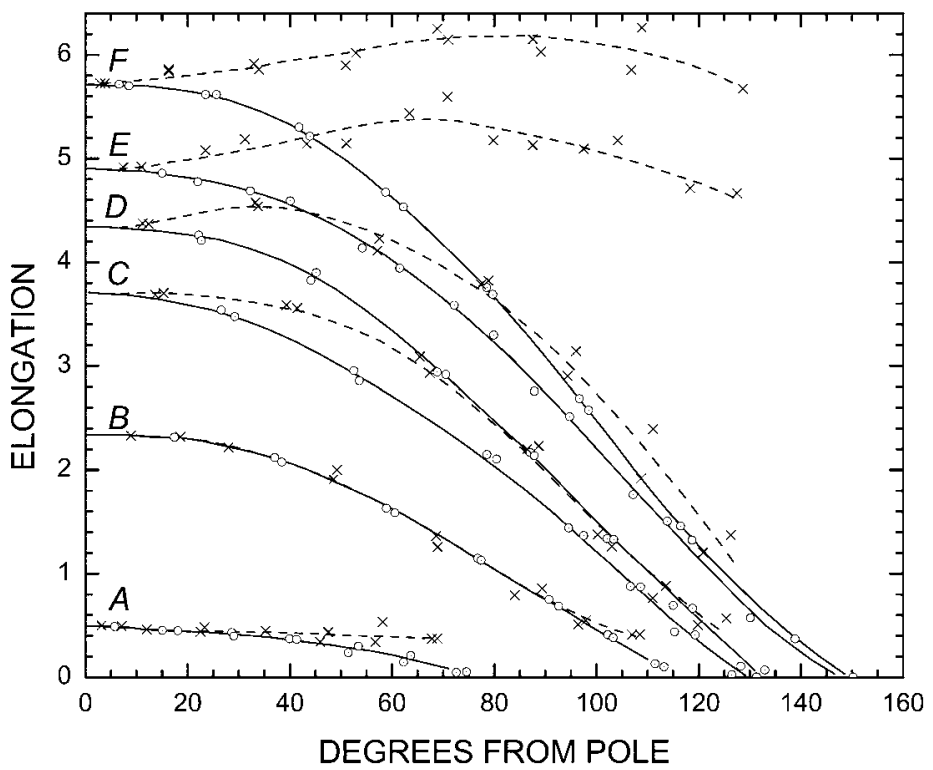

FIG. 1. - Experimental strains reported by $\operatorname{Treloar}^{4}$ (radial, $\times x \times$; circumferential, $\left.\bigcirc \bigcirc \bigcirc\right)$, along with his fits (radial, --- circumferential, - . See text for explanation of the abscissa.

The lowest inflation in Figure 1 (curve A) gives a biaxial strain of about 50\%, which is near the limit of sensitivity. The large scatter makes any conclusions uncertain, even though the strains for many practical applications fall below this. Data for the higher inflations in Figure 1 are more reliable, and indicate that a change in the nature of the deformation occurs when the pole biaxial strain is about 4.5. Below this value (curves B, C, and D), the radial and the circumferential deformations track closely; that is, the strain is nearly equi-biaxial (i.e., $\varepsilon_{C} \approx \varepsilon_{R}$ ), over most of the disk. On the other hand, when the pole strain exceeds 4.5 (curves $E$ and $F$ ), $\varepsilon_{R}$ is nearly constant, while $\varepsilon_{\mathrm{C}}$ decreases to zero at the edge. Thus, inflation induces a state of pure shear $\left(\varepsilon_{\mathrm{R}}\right.$ " $\varepsilon_{C}$ ) over most of the disk. This intriguing result, a strain-dependent deformation mode, requires explanation.

There have since been a number of analytical investigations of inflated rubber membranes. Adkins and Rivlin ${ }^{1}$ employed a Taylor series expansion of the principal extension ratios, to obtain a numerical solution for the principal stresses and strains. They predicted that different strain energy functions, such as the Mooney-Rivlin and neo-Hookean forms, will produce markedly different strain distributions. Subsequently, Hart-Smith and Crisp ${ }^{11}$ utilized a more complicated strain energy function to obtain better agreement with Treloar's experimental data, while Oden ${ }^{12}$ employed the finite element method. Haddow et al. ${ }^{13,14}$ recast the problem in terms of Lagrangian equilibrium equations, expressed in terms of Biot stresses. Vaughan ${ }^{15}$ accounted for prestretch of the membrane, and Wineman and Huntley ${ }^{16}$ included damage-induced softening. Taber ${ }^{17}$ investigated the transition to thick membranes. Recently, Hsu et al. ${ }^{18}$ converted the problem to two-dimensional elasticity.

Unfortunately, experimental verification of these theoretical studies is lacking. Feng ${ }^{19}$ measured the pressure and pole height of a relaxing, inflated rubber sheet. He compared the results to a viscoelastic model, but strains were measured only at the pole. Przybylo and Arruda ${ }^{20}$ published experimental profiles of inflated rubber sheets, comparing finite element calculations employing different constitutive models; however, the distribution of strains was not examined. 
Charrier et al. ${ }^{21}$ investigated inflation in membranes with various geometries, but no information was obtained concerning the strain distributions for the reported profiles. Similarly, Hsu et al. ${ }^{22}$ determined average strains in soft biological membranes, but not strain distributions. Biaxial inflation has also been employed to determine the strain energy function, ${ }^{23,24}$ but attention in these studies focussed on the strain at the pole. To our knowledge, only Treloar ${ }^{4}$ has published experimental data concerning the position-dependence of the strain in biaxially-deformed rubber. Moreover, as mentioned above, Treloar's most accurate results are at large strains.

\section{EXPERIMENTAL}

Deproteinized natural rubber (SMR-S from H. A. Astlett Co.) was mixed on a laboratory mill with $2.8 \mathrm{phr}$ dicumyl peroxide and $1.0 \mathrm{phr}$ of a dihydroquinoline antioxidant. Films of approximately $0.9 \mathrm{~mm}$ thickness were compression molded at $160{ }^{\circ} \mathrm{C}$ for 60 minutes. Taking care that the thickness was uniform, fiducial marks (in the form of a fine grid of circular and radial lines) were printed on a selected region of the film, using a rubber stamp. A circular sample, diameter $120 \mathrm{~mm}$, was cut from this film.

The outer edge of the sample was lightly clamped into position. The clamping pressure squeezed a small amount of rubber from beneath the flange, causing slight buckling, estimated to be equivalent to $2 \%$ compression. Inflation of the film with nitrogen was accomplished by displacing a piston inside a cylinder. The pressure was measured via an Omega PX236-005 transducer. After inflation, the pressure would decrease $c a .5 \%$ in ten minutes, and thereafter remain constant; the latter was taken as the equilibrium value. Repeated inflation cycles gave identical pressures.

The (true) stress at the pole was found from ${ }^{1}$

$$
\sigma_{t}=P \lambda^{2} \frac{R}{2 t_{0}}
$$

where $P$ is the pressure; $\lambda$ the (biaxial) stretch ratio at the surface; and $t_{0}$ the undeformed film thickness. The radius of curvature, $R$, was determined by measuring the vertical rise of the pole. The strain $\varepsilon(=\lambda-1)$ was determined from photographs of fiducial marks, as described below.

In addition to biaxial inflation, conventional tensile stress-strain measurements were carried out (Instron 4206 with Wallace optical extensometer) on strips $(90 \times 12.7 \mathrm{~mm})$ cut from the same cured sheet. At the slow strain rate employed, $0.00167 \mathrm{~s}^{-1}\left(0.1 \mathrm{~min}^{-1}\right)$, the extension and retraction curves were virtually identical, whereby the retraction data were taken to be equilibrium. To avoid strain-induced crystallization, the maximum extension was limited to $100 \% .^{25}$

A finite element method ${ }^{26}$ was implemented using ABAQUS. The membrane was discretized using 100 3-node axi-symmetric shell elements, each employing a quadratic interpolation function. Translational displacements associated with the node on the membrane periphery were fixed, and a specified volume of fluid introduced to obtain the desired strain at the pole. No attempt was made to model localized effects associated with the clamp or buckling.

\section{IMAGE CALIBRATION}

In the pole stress-strain measurements, the calibration of fiducial displacements was made by including a ruler in the photograph of the inflated sheet, held touching the rubber near the pole. The stretch was determined over a small area about the pole, from an average of eight displacement measurements. However, this procedure proved to be inadequate for larger sections, because of scaling changes due to (1) changes in the perspective with the viewing distance and (2) the continually changing angle between the surface normal and the view vector. Particularly 
at low inflation, accurate results required a better calibration.

A representative photograph of the inflated sheet is shown in Figure 2, where the strain at the pole was approximately $26 \%$. The ring clamp is at the right edge, labeled " $\mathrm{A}$ ". The dark lines, concentric to the ring clamp, are the latitude fiducial lines; the lines perpendicular to these are the longitude lines. The longitude lines all meet at the pole, out of view, to the left of the picture. The pixel positions of the intersecting lines were digitized, and these points are indicated by circles, and superimposed on the photograph. To provide the scale, a section of graph paper was prepared with a rectangular hole, and affixed to the inflated membrane with a small amount of silicone grease. This calibration appears in Figure 2 around the outer edges. Points on this scale were likewise digitized, and are indicated by squares. The distance between the graph paper lines was $1.27 \mathrm{~mm}$.

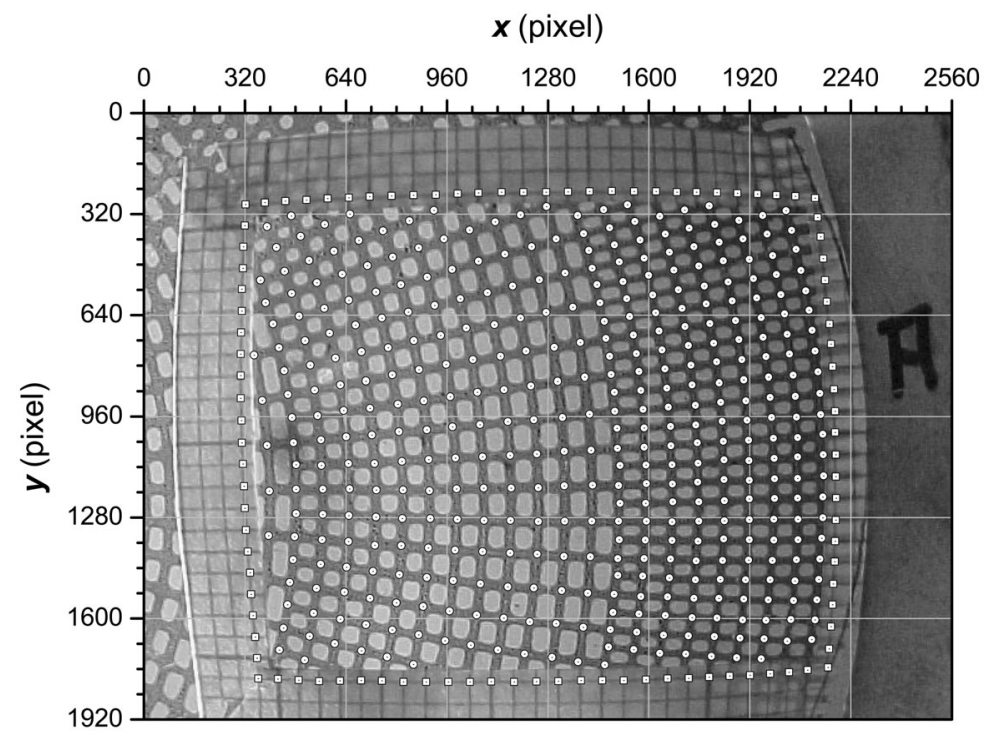

FIG. 2. - Photograph of sheet inflated to $26 \%$ biaxial strain at the pole. The superimposed circles represent the position of the digitized points on the sheet, and the squares along the border are the digitized positions of the scale.

Let $\left(X_{\mathrm{i}}, Y_{\mathrm{i}}\right)$ be the actual position of $i$, as defined by the graph paper scale on the periphery of the photograph, and $\left(x_{\mathrm{i}}, y_{\mathrm{i}}\right)$ be its corresponding pixel position. Focussing on the graph scale along the bottom of Figure 2, the function $\partial X_{\mathrm{i}} / \partial x_{\mathrm{i}}$ defines the pixel spacing between the lines, in the horizontal direction. This function is likewise defined by the scale row across the top of Figure 2. The overall spacing along the $x$ direction, which also changes as a function of $y$, is found from the horizontal distance between the corresponding vertical scale, at the left and right edges. This overall spacing is an average over the entire width, denoted by $\overline{\mathrm{x}}$. The data from for both scales, $\partial X_{\mathrm{i}} / \partial x_{\mathrm{i}}$ and $\overline{\mathrm{x}}$, were well described by parabolas, as were $\partial Y_{\mathrm{i}} / \partial y_{\mathrm{i}}$ and $\overline{\mathrm{y}}$. The fits of the scale data $\partial X_{\mathrm{i}} / \partial x_{\mathrm{i}}$ and $\partial Y_{\mathrm{i}} / \partial y_{\mathrm{i}}$ were employed to calculate the actual positions $\left(X_{\mathrm{i}}, Y_{\mathrm{i}}\right)$, from

$$
X_{i}\left(x_{i}, y_{i}\right)=\frac{\bar{X}(y)}{\bar{X}_{0}} \int \frac{\partial X}{\partial x} d x
$$

and 


$$
Y_{i}\left(x_{i}, y_{i}\right)=\frac{\bar{Y}(y)}{\bar{Y}_{0}} \int \frac{\partial Y}{\partial y} d y
$$

where $\bar{x}_{0}$ is the average horizontal spacing at the upper and lower edges, and $\bar{y}_{0}$ is the average vertical spacing at the left and right edges.

Figure 3 compares the pixel positions $\left(x_{\mathrm{i}}, y_{\mathrm{i}}\right)$, shown as filled squares, to the actual positions $\left(X_{\mathrm{i}}, Y_{\mathrm{i}}\right)$, shown as open circles. The application of Equations 2 and 3 have straightened the curved lines of the edge scales. From data set $\left(X_{\mathrm{i}}, Y_{\mathrm{i}}\right)$, we found the radial and circumferential strains, $\varepsilon_{\mathrm{R}}$ and $\varepsilon_{\mathrm{C}}$, from the displacements in the longitude and latitude directions, respectively.

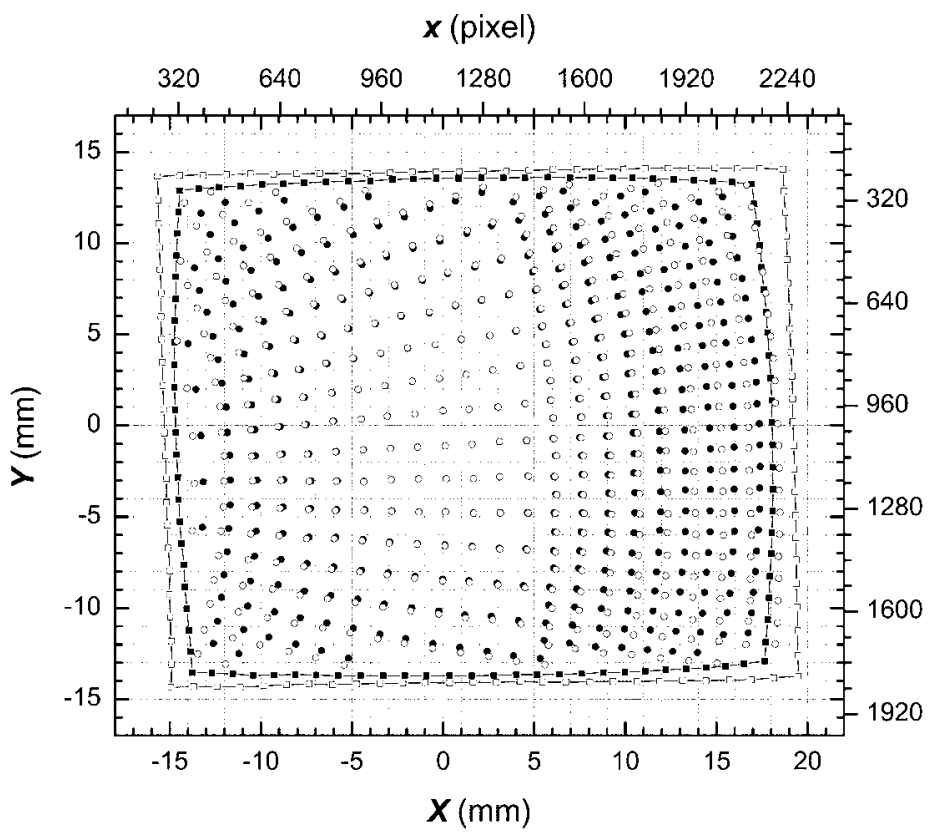

FIG. 3. - Results of correcting the data in Figure 2 for parabolic calibration. Original pixel positions of the points and the scale are given as filled circles and squares, respectively. Superimposed on these are the corrected positions (open circles and squares, respectively). Note that the correction has straightened the originally curved scale at the border.

\section{RESULTS AND DISCUSSION}

The stress-strain curve, plotted in the Mooney-Rivlin ${ }^{27-30}$ form of reduced stress $f(=$ $\sigma /\left(\lambda-\lambda^{-2}\right)$, where $\sigma$ is the engineering stress) as a function of $1 / \lambda$, is shown in Figure 4. The maximum extension was equal to 2 , yielding linear behavior for much of the range, $2.5>1 / \lambda \geq 0.5$. Thus, the Mooney-Rivlin equation,

$$
f=2\left(C_{1}+\frac{C_{2}}{\lambda}\right)
$$

where $C_{1}$ and $C_{2}$ are constants, is adequate for the low strain levels of interest. Adkins and Rivlin ${ }^{1}$ also found this form to be in good agreement with experimental measurements on natural rubber. However, as seen in Figure 4, at larger compressive strains ( $\geq 60 \%$ ), Equation 4 substantially overestimates the modulus. This behavior is consistently seen in gum rubbers. ${ }^{23,24,31-33}$ 
Better accuracy can be realized using a general form of the strain energy function suggested by Rivlin. ${ }^{22}$ The curvature in the stress-strain response is captured to some extent by molecular theories of rubber deformation, such as the constraint models, ${ }^{34-40}$ which include the effect of topological interactions on the microscopic deformation. However, these models still tend to overestimate the stress for compression. ${ }^{31-33}$

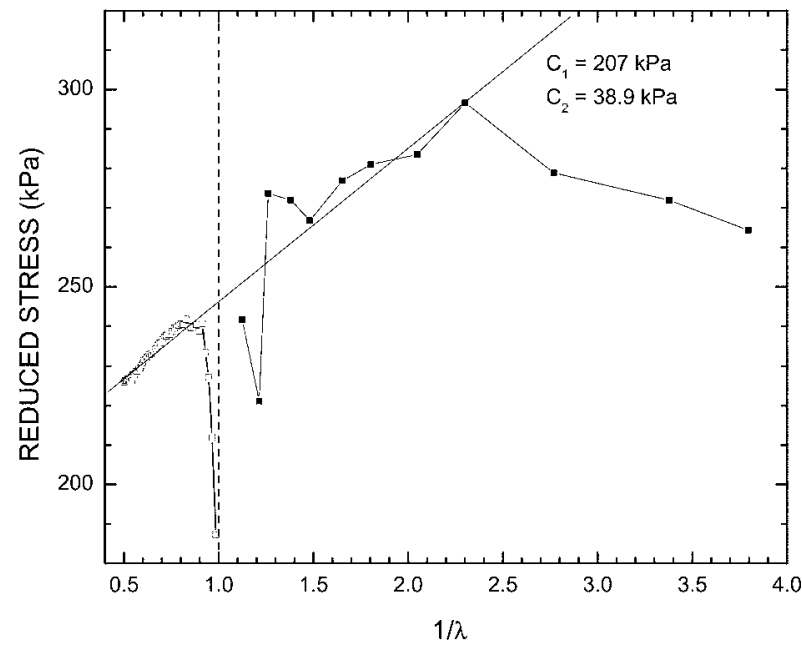

FIG. 4. - Equilibrium stress-strain data for the gum natural rubber. The solid line is the fit to the Mooney-Rivlin function, using the indicated coefficients. The deviations in the vicinity of $\lambda=1$ reflect the nature of the fitting function (Equation 4). At high compressive strain $(\lambda<0.4)$, the Mooney-Rivlin description overestimates the stress.

The measured strain distributions for three separate inflations, nominally $12 \%, 26 \%$, and $33 \%$ biaxial strain at the pole, are displayed as points in Figure 5. The strain is equi-biaxial up to a normalized radius value of about 0.2 . The circumferential strain falls to zero at the clamp, while the radial strain remains nearly constant throughout, producing a state of pure shear at the edge.

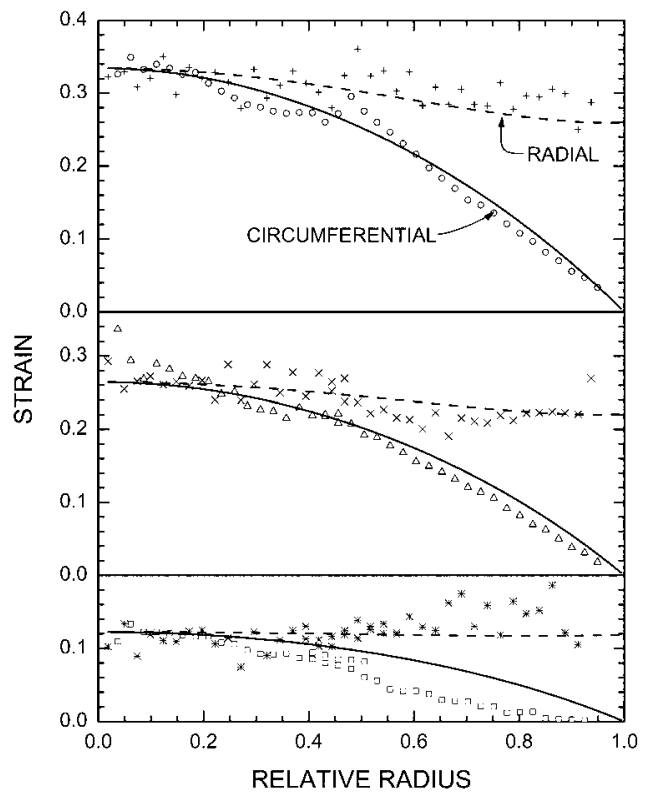

FIG. 5. - Experimentally determined strains as a function of radial position.

The solid and dashed lines are the finite element results. 
The finite element results, generated with the Mooney-Rivlin coefficients from Figure 4, are displayed as smooth lines in Figure 5. The agreement with the measured data is within the experimental scatter. At the low strains associated with the present experiments, the finite element calculations are relatively insensitive to the quantity $C_{2} / C_{1}$, and the radial strains decrease monotonically with radius. At higher strains, the dependence on this ratio increases. As the pole strain increases, the maximum radial strain does not occur at the pole, but shifts towards the clamp.

Most significant is the limited range of the equi-biaxial region in Figure 5. This is quite different from Treloar's ${ }^{4}$ results at moderate, but somewhat higher strains (curves B, C, and D of Figure 1), which show essentially equi-biaxial strain over most of the membrane. On the other hand, the data in Figure 5 roughly agree with the lowest strain data of Figure 1 (curve A), notwithstanding the sensitivity limit. Unfortunately, there is insufficient detail in Reference 4 for a quantitative comparison.

Strain-crystallization of the natural rubber can exert an influence. Although the strains in the present experiments were too small, it is likely that crystallization transpires during Treloar's measurements at higher strains. Since the strain energy function used in previous modeling efforts ${ }^{1,11-20,22}$ excludes this effect, discrepancies between theoretical predictions and Treloar's results may be expected for higher strains.

\section{CONCLUSIONS}

The strain distribution in an inflated sheet of natural rubber was measured for biaxial strains as high as $33 \%$. Only near the pole region is a state of equal biaxial deformation attained. While the radial strain is nearly independent of position, the circumferential strain monotonically decreases to zero with proximity to the edge. This gives rise to planar extension (pure shear) in the vicinity of the clamp. These results depart from previously published measurements of Treloar, ${ }^{4}$ which suggested that at lower strain, biaxial deformation persists throughout the sheet. While the reason for this discrepancy is unclear, we emphasize that our sensitivity is much better than Treloar's, and that our measurements are corroborated by finite element calculations. Our results are consistent with Treloar's higher strain data, indicating that there is no discontinuity in strain distribution as the degree of inflation increases. Of course, this does not consider the elastic instabilities observed at very high degrees of inflation. ${ }^{41}$

A finite element model of the position-dependent strain gave a good fit to the measured data. Thus, at least for moderate strains, the Mooney-Rivlin equation can adequately describe the material response, notwithstanding its well-known deficiencies. ${ }^{23,24,31-33}$ Since the mode of deformation governs the connection between the strain energy and the amount of energy available to propagate cracks in rubber, the strain distribution can exert a dominating effect both on the fatigue lifetime and on the locus of failure. Thus, the present results are relevant to analysis of the failure properties of rubber subjected to inflation and related biaxial strains.

\section{ACKNOWLEDGEMENTS}

The work at NRL was supported by the Office of Naval Research. We thank C. G. Robertson, who constructed the biaxial inflation device. This work originated from discussions with G. R. Hamed.

\section{REFERENCES}

${ }^{1}$ J. E. Adkins and R. S. Rivlin, Philosoph. Trans. R. Soc. London, Series A 244, 505 (1952).

${ }^{2}$ A. D. Shah and J. D. Humphrey, J.Biomech. 32, 593 (1999).

${ }^{3}$ J. D. Humphrey and P. B. Canham, J. Elasticity 61, 49 (2000). 
${ }^{4}$ L. R. G. Treloar, Trans. Instit. Rubber Ind. 19, 201 (1944).

${ }^{5}$ B. J. Roberts and J. B. Benzies, Proc. Rubbercon 77, 2.1 (1977).

${ }^{6} \mathrm{~J}$. F. Roach, Ph.D. Thesis, University of Akron, 1982.

${ }^{7}$ J. Little, Undersea Warfare 1, 12 (1999).

${ }^{8}$ I. S. Choi, C. M. Roland, and L. C. Bissonnette, Rubber Chem. Technol. 67, 892 (1994).

${ }^{9}$ P. H. Mott and C. M. Roland, Rubber Chem. Technol. 74, 79 (2001).

${ }^{10}$ C. M. Roland and I. S. Choi in "Rubber Technology International,” L. Gale, ed., UK \& International Press, 1996, p. 12.

${ }^{11}$ L. J. Hart-Smith and J. D. C. Crisp, Inter. J. Engi. Sci. 5, 1 (1967).

${ }^{12}$ J. T. Oden, "Finite Elements of Nonlinear Continua," New York: McGraw Hill, 1972, ch. 18.

${ }^{13}$ J. B. Haddow, J. L. Wegner, and L. Jiang, Acta Mech. 92, 77 (1992).

${ }^{14}$ L. Jiang and J. B. Haddow, Comput. Struct. 57, 401 (1995).

${ }^{15}$ H. Vaughan, Q. J. Mech. Appl. Math. 34, 111 (1981).

${ }^{16}$ A. S. Wineman and H. E. Huntley, Inter. J. Solids Struc. 31, 3295 (1994).

${ }^{17}$ L. A. Taber, J. Appl. Mech. - Trans. ASME 54, 578 (1987).

${ }^{18}$ F. P. K. Hsu, C. Schwab, D. Rigamonti, and J. D. Humphrey, Inter. J. Solids Struct. 31, 3375 (1994).

${ }^{19}$ W. W. Feng, J. Appl. Mech. 59, S29 (1992).

${ }^{20}$ P. A. Przybylo and E. M. Arruda, Rubber Chem. Technol. 71, 730 (1998).

${ }^{21}$ J.-M. Charrier, S. Shrivastava, and R. Wu, J. Strain Anal. 24, 55 (1989).

${ }^{22}$ F. P. K. Hsu, A. M. C. Liu, J. Downs, D. Rigamonti, and J. D. Humphrey, IEEE Trans. Biomed. Eng. 42, 442 (1995).

${ }^{23}$ R. S. Rivlin and D. W. Saunders, Philos. Trans. R. Soc. London Ser. A 243, 251 (1951).

${ }^{24}$ P. Xu and J. E. Mark, J. Polym. Sci.: Part B: Polym. Phys. 29, 355 (1991).

${ }^{25}$ I.S. Choi and C.M. Roland, RubBer Chem. TeChNOL. 70, 202 (1997).

${ }^{26}$ J. T. Oden, op. cit., chapter 15.

${ }^{27}$ M. Mooney, J. Appl. Phys. 11, 582 (1940).

${ }^{28}$ M. Mooney, J. Appl. Phys. 19, 434 (1948).

${ }^{29}$ R. S. Rivlin, Philos. Trans. R. Soc. London Ser. A 240, 459 (1948).

${ }^{30}$ R. S. Rivlin, Philos. Trans. R. Soc. London Ser. A 241, 379 (1948).

${ }^{31}$ P. H. Mott and C. M. Roland, Macromolecules 29, 6941 (1996).

${ }^{32}$ C. M. Roland, P. H. Mott, and G. Heinrich, Comput. Theoret. Polym. Sci. 9, 197 (1999).

${ }^{33}$ C.M. Roland and P.H. Mott, Macromolecules 31, 4033 (1998).

${ }^{34}$ P. J. Flory, J. Chem. Phys. 66, 5720 (1977).

${ }^{35}$ P. J. Flory and B. Erman, Macromolecules 15, 800 (1982).

${ }^{36}$ A. Kloczkowski, J. E. Mark, and B. Erman, Macromolecules 28, 5089 (1995).

${ }^{37}$ A. Kloczkowski, J. E. Mark, and B. Erman, Comput. Polym. Sci. 5, 37 (1995).

${ }^{38}$ G. Heinrich, E. Staube, and G. Helmis, Adv. Polym. Sci. 85, 33 (1988).

${ }^{39}$ M. Gottlieb and R. J. Gaylord, Macromolecules 20, 130 (1987).

${ }^{40}$ M. Kaliske and G. Heinrich, RubBer CHEM. TeCHnOL. 72, 602 (1999).

${ }^{41}$ A. N. Gent, Rubber Chem. Technol. 72, 263 (1999). 